Casos Clínicos

Arch. Esp. Urol. 2010; 63 (7): 559-562

\section{PRIAPISMO RECURRENTE ("STUTTERING PRIAPISM"): PRESENTACIÓN DE UN NUEVO CASO Y REVISIÓN DE LA LITERATURA}

Roberto Molina Escudero, Elena Rodríguez Fernández, Enrique Lledó García, José Jara Rascón, Juan Tabares Jiménez, Adrian Husillos Alonso y Carlos Hernández Fernández.

Servicio Urología. Hospital Universitario Gregorio Marañón. Madrid. España.

Resumen.- OBJETIVO: Revisar la forma de presentación, fisiopatología, diagnostico y alternativas terapéuticas del priapismo recurrente mediante la presentación de un nuevo caso.

MÉTODOS: Varón de 25 años, estudiado en otro centro por presentar episodios recurrentes de priapismo desde hace aproximadamente 18 meses. Estos episodios se producen a diario, interfiriendo de forma importante con la calidad de vida del paciente.

RESULTADOS: Se inicio tratamiento con Bicalutamida $50 \mathrm{mg} / 24 \mathrm{~h}$ sin mejoría. En la analitica, ecografía doppler

CORRESPONDENCIA

Roberto Molina Escudero

Servicio Urología

Hospital General Universitario Gregorio

Marañón

Doctor Esquerdo, 46

28007 Madrid. (España)

robersescu@hotmail.com

Aceptado para publicar: 14 de noviembre 2009. peneana y arteriografía selectiva de arterias pudendas no se evidenciaron alteraciones. Se pautó Tadalafilo 5mg/ $24 \mathrm{~h}$ durante dos meses sin respuesta. Posteriormente se instauró tratamiento con Diazepam $10 \mathrm{mg} / 24 \mathrm{~h}$ y Terbutalina $5 \mathrm{mg} / 24 \mathrm{~h}$ permitiendo el control de la enfermedad, quedando asintomático en la actualidad.

CONCLUSIONES: El priapismo recurrente es una forma poco común de presentación de esta enfermedad, producida por una alteración en los mecanismos de regulación de la erección mediados por la 5PDE y el GMPC.

Se han propuesto varios fármacos en su tratamiento con eficacia variable, aunque no existen series suficientemente largas para poder recomendar ninguno como primera opción. El uso de inhibidores de la 5PDE de forma prolongada, ha sido utilizado con éxito por algunos grupos.

El conocimiento de estas alternativas, es importante para el tratamiento de esta compleja e infrecuente patología.

Palabras clave: Priapismo recurrente. Inhibidores de la 5PDE.

Summary.- OBJECTIVE: To review the presentation, physiopathology, diagnosis and therapeutic alternatives of stuttering priapism with the contribution of a new clinical case.

METHODS: A 25 year old man, studied in another center for recurrent episodes of priaprism for about 18 months. These episodes occur daily, significantly interfering with patient's quality of life.

RESULTS: Initially he was treated with Bicalutamide $50 \mathrm{mg} /$ 24 h with no improvement. Blood test, penile Doppler ultrasound and selective arteriography of pudendal arteries showed no abnormalities. Tadalafil $5 \mathrm{mg} / 24 \mathrm{~h}$ was given for two months without response. Subsequently were treated with Diazepam $10 \mathrm{mg} / 24 \mathrm{~h}$ and Terbutaline $5 \mathrm{mg} / 24 \mathrm{~h}$ allowing control of the disease, remaining asymptomatic at present.

CONCLUSIONS: Stuttering priapism is a rare form of presentation of this disease, caused by an alteration in the regulatory mechanisms of erection mediated by SPDE and cGMP.

Several drugs have been proposed in treatment with variable effectiveness, though there is no series long enough to recommend either as first choice. The use of inhibitors 5PDE so long, has been used successfully by some groups.

Knowledge of these alternatives is important for the treatment of this complex and unusual pathology.

Keywords: Stuttering priapism. 5PDE inhibitors. 


\section{INTRODUCCIÓN}

El priapismo se define como una erección persistente, involuntaria, en ausencia de estimulo o deseo sexual, con una duración superior a 4-6 horas.

Este término procede del dios griego Priapus, dios de la fertilidad, cuyo gran falo era símbolo de la masculinidad. Encontramos papiros egipcios que hablan de esta patología y proponen tratamientos frente a la misma.

La primera publicación a cerca del priapismo fue realizada en el año 1616 por Petraens, siendo en 1824 cuando Callaway describió el primer caso en lengua inglesa (1).

Su incidencia se encuentra en torno a $1 / 100.000$ habitantes, habiéndose incrementado en los últimos años por la generalización del uso de inyecciones intracavernosas e inhibidores de la 5 fosfodiesterasa (5PDE) en el tratamiento de la disfunción eréctil (2).

Desde el punto de vista etiológico existen múltiples causas (neoplasias hematológicas, traumatismos, drogas, picaduras de insecto...), siendo la más frecuente $(50 \%)$ la idiopática.

Tradicionalmente el priapismo se clasifica en tres formas: isquémico, arterial y recurrente.

El isquémico (bajo flujo), es el más frecuente, y se caracteriza por la ausencia de flujo sanguíneo cavernoso que condiciona una erección prolongada, generalmente dolorosa con participación del glande y del cuerpo esponjoso. En la gasometría cavernosa se evidencia acidosis, hipercapnia e hipoxia. Constituye una emergencia médica por el riesgo de necrosis y fibrosis del cuerpo cavernoso que conlleve a una disfunción eréctil permanente.

La variante no isquémica (arterial o de alto flujo), suele estar producida por una fístula arterio-cavernosa secundaria a traumatismos y se caracteriza por una erección menos marcada, no dolorosa y con gasometría cavernosa normal, no constituyendo una emergencia.

Por último existe una forma poco común, la variante recurrente ("Stuttering priapism"), que consiste en episodios de priapismo que acontecen generalmente durante el sueño, con tumescencia normal entre las crisis.

\section{CASO CLÍNICO}

Varón de 25 años estudiado en otro centro por presentar episodios recurrentes de priapismo desde hace aproximadamente 18 meses.

En su historia clínica no se encuentran antecedentes médico-quirúrgicos de interés, ni uso de sustancias de abuso, así como ningún evento precipitante reconocible.

El paciente refiere que inicialmente los episodios de priapismo acontecían $1 \mathrm{vez} / \mathrm{mes}$, necesitando destumescencia con alfa adrenérgicos intracavernosos en el servicio de urgencias en varias ocasiones. Seis meses después, el cuadro se repite de forma diaria hasta la actualidad, interfiriendo de un modo muy importante en sus actividades cotidianas, en su vida de pareja y profesionalmente.

Las crisis consisten en erecciones nocturnas dolorosas, que despiertan al paciente, caracterizadas por la turgencia e hipersensibilidad del glande y la participación del cuerpo esponjoso, no cediendo tras la eyaculación.

Los episodios ceden mediante la realización de ejercicio físico intenso durante un periodo mínimo de 1 hora, aunque ocasionalmente el paciente necesita acudir a urgencias para conseguir la destumescencia.

En la valoración inicial se realizó exploración física y analítica completa, descartándose neoplasias hematológicas y anemia de células falciformes como agentes etiológicos. Se inició tratamiento con Bicalutamida $50 \mathrm{mg} / 24 \mathrm{~h}$ durante dos meses sin mejorar la sintomatología.

Ante la persistencia de los síntomas el paciente es remitido a nuestro centro donde se realizan eco doppler peneano y arteriografía selectiva de las arterias pudendas; descartándose fístulas arterio-cavernosas.

Con el diagnostico de priapismo recurrente idiopático se inicia tratamiento con Tadalafilo $5 \mathrm{mg} /$ día durante tres meses, sin éxito.

Tras el fracaso terapéutico inicial, se propuso al paciente el uso combinado de Terbutalina $5 \mathrm{mg} / 24 \mathrm{~h}$ y Diazepam $10 \mathrm{mg} / 24 \mathrm{~h}$ durante tres meses, consiguiendo el control de la enfermedad, encontrándose el paciente asintomático en la actualidad.

\section{DISCUSIÓN}

El priapismo recurrente es la forma menos común de presentación de esta enfermedad. Se caracteriza por la presencia de erecciones dolorosas nocturnas de repetición, generalmente de corta duración, ocasionalmente más prolongadas, precisando atención médica urgente. Estos pacientes presentan una función sexual normal en el intervalo intercrítico. Los episodios pueden ser de alto o bajo flujo.

Las causas más frecuentes de esta patología son la anemia de células falciformes y las neoplasias hematológicas, aunque existe un amplio porcentaje de casos 
donde no se evidencia ninguna patología subyacente, definiéndose como idiopático.

Fisiopatologicamente se caracteriza por una alteración en la síntesis de oxido nítrico (NO) por parte del endotelio del músculo liso cavernoso. Esto condiciona una disminución de la expresión de una proteína kinasa dependiente de GMPc, que secundariamente produce una disminución de los niveles de la 5PDE.

Como consecuencia de esta alteración de la regulación del músculo liso cavernoso se produce una respuesta alterada a los estímulos sexuales (nocturnos, reflexógenos, o psicógenos), dando como resultado una erección anormalmente prolongada (3).

El diagnostico se establece mediante la historia de erecciones nocturnas dolorosas recurrentes, siendo necesario descartar mediante determinación analítica sanguínea, leucemias, linfomas y anemia de células falciformes como responsables del cuadro clínico. Otras exploraciones complementarias como eco-doppler de los cuerpos cavernosos, o arteriografía están indicadas para descartar fístulas arterio-cavernosas, antes de considerar el priapismo como idiopático.

En relación al tratamiento, existen múltiples opciones, aunque ninguna de ellas se puede considerar de elección dada la escasa información existente en la literatura, siendo la mayoría de ellos series muy cortas o casos clínicos aislados (4).

El uso de antiandrógenos y análogos de LHRH es el tratamiento clásico, con una aceptable tasa de respuestas, pero con un alto índice de disminución de la libido y atrofia testicular, como demuestran Dahm et al. en una serie de nueve pacientes (5).

La utilización de Digoxina a dosis de 0.25-0.50 mg/ día fue propuesta por Sadeghi-Nejad et al en un estudio de Digoxina vs placebo. Este autor demostró un incremento de la concentración de calcio en el músculo liso cavernoso, disminuyendo la frecuencia de las erecciones con mínimos efectos sobre la función androgénica (6).

La Terbutalina oral a dosis de 5-10 mg diarios también se ha utilizado para disminuir las recurrencias gracias a su efecto agonista beta adrenérgico. Lowe et al realizaron un estudio comparando la eficacia de Terbutalina vs placebo en 75 pacientes con priapismo recurrente, obteniendo una respuesta del $36 \%$ (7). En otro estudio con 68 pacientes, Priyadarschi et al compararon de nuevo la eficacia de Terbutalina vs placebo en el tratamiento de esta patología, obteniendo una tasa de respuestas del $42 \%$ en los pacientes tratados con Terbutalina, frente al $15 \%$ de que recibieron placebo (8).

Otro agente oral utilizado es el Baclofeno, un agonista del receptor GABA que produce una disminución de los reflejos mono y polisinápticos a nivel espinal. La expe- riencia en su uso se limita a pequeñas series de casos clínicos (9).

La utilización de otros fármacos como Gabapentina, $\mathrm{Hi}$ dralacina, o Hidroxiurea esta descrita en casos clínicos aislados.

Recientemente se ha postulado el uso de inhibidores de la 5PDE a bajas dosis de forma continua en el tratamiento del priapismo recurrente. Este uso permite una regulación adecuada de los elementos que intervienen en el proceso de la erección: 5PDE y GMPc, fundamentalmente, basado en la teoría de una alteración en la expresión de este enzima como responsable del cuadro clínico, aunque si bien es cierto que este uso de los inhibidores de la 5PDE es controvertido (10).

La disregulación de la 5PDE en pacientes con deficiencia de la Óxido Nítrico Sintetasa endotelial provoca una supersensibilización del tejido eréctil al GMPc, originando una respuesta eréctil desproporcionada a estímulos psicógenos y reflexógenos tales como la fase REM del sueño o manipulación genital (11).

Existen otras alternativas terapéuticas de uso menos extendido como es la aplicación intracavernosa de fenilefrina por el propio paciente $u$ otras aminas simpaticomiméticas.

En los casos refractarios al tratamiento médico, los shunts corporo cavernosos y el implante de una prótesis de pene pueden considerarse como una alternativa terapéutica (12).

Es importante realizar el diagnostico diferencial con las erecciones dolorosas relacionadas con el sueño. Estas se caracterizan por presentar una alteración de la función autónoma durante el sueño, teniendo como resultado dolor en las erecciones que aparecen durante la fase R.E.M. despertando al paciente y cediendo generalmente con medidas físicas. El tratamiento consiste en la administración de antidepresivos como amitriptilina, clozapina o paroxetina en dosis única diaria antes de dormir (13).

\section{CONCLUSIONES}

El priapismo recurrente es una forma poco común de presentación de esta enfermedad, en la que se produce una alteración en los mecanismos de regulación de la erección mediados por la 5PDE y el GMPc, teniendo como consecuencia la presencia de erecciones dolorosas nocturnas que pueden ser de alto o bajo flujo.

Se han propuesto varios fármacos en su tratamiento con eficacia variable, aunque no existen series suficientemente largas para poder recomendar ninguno como primera opción. Paradóiicamente el uso de inhibidores de la 5PDE de forma prolongada, ha sido utilizado con 
éxito por algunos grupos, constituyendo una opción terapéutica adicional.

El conocimiento de estas alternativas, es importante para el urólogo, de cara al tratamiento de esta compleja e infrecuente patología.

\section{BIBLIOGRAFIA y LECTURAS RECOMENDADAS (*lectura de interés $y^{* *}$ lectura fundamental)}

1. Callaway T. Unusual case of priapism. London Medical Repository 1824; 1:286,

*2. Rodríguez R, García S, Puigvert A, Pomerol J.M, Munárriz R. Priapismo. Actas Urol Esp, 2005; 29: 961-8.

*3. Yuan J, DeSouza R, Westney L, Wang R. Insights of priapism mechanism and rationale treatment for recurrent priapism. Asian J Androl, 2008; 10: 88-101.

4. Muneer A, Minhas S, Arya M, Ralph J. Stuttering priapism - a review of the therapeutic options. Int J Clin Pract, 2008; 62:1265-70.

5. Dahm P, Rao DS, Donatucci CF. Antiandrogens in the treatment of priapism. Urol, 2002; 59: 138.
6. Sadeghi-Nejad H, Gupta S, Salimpour P, Daller M, Krane RJ, Goldstein I. Treatment of recurrent venoocclusive priapism by digoxin. J Urol, 1997; 157: 202.

*7. Lowe FC, Jarow JP. Placebo-controlled study of oral terbutaline and pseudoephedrine in management of prostaglandin E1-induced prolonged erections. Urol, 1993; 42: 51-3.

8. Priyadarshi S. Oral terbutaline in the management of pharmacologically induced prolonged erection. Int $\mathrm{J}$ Impot Res 2004; 16: 424-6.

9. Rourke KF, Fischler AH, Jordan GH. Treatment of recurrent idiopathic priapism with oral baclofen. $\mathrm{J}$ Urol, 2002; 168: 2552-3.

**10. Burnett A, Trinity J, Champion H, Musicki B. Longterm oral phosphodiesterase 5 inhibitor therapy alleviates recurrent priapism. Urol, 2006; 67:1043-8.

11. Champion HC, Bivalacqua TJ, Takimoto E, Kass DA, Burnett AL. Phosphodiesterase-5A dysregulation in penile erectile tissue is a mechanism of priapism. Proc Natl Acad Sci USA, 2005; 102: 1661-6.

12. Carson C. Surgery for Stuttering Priapism. J Urol, 2009; 181:449-51.

13. Menéndez V, Mora A, Prieto L, Galan J, Fernandez C, García F. Erecciones dolorosas relacionadas con el sueño. Actas Urol Esp, 1999; 23: 539-41. 